\title{
ARTICLE OPEN Witnessing the quantumness of a system by observing only its classical features
}

\author{
C. Marletto ${ }^{1}{ }^{1}$ and V. Vedral ${ }^{1,2}$
}

A central problem in fundamental physics is to witness the non-classicality of systems whose dynamics is not completely specified. For example, this arises in testing predictions that a macroscopic system, such as a living system, obeys quantum theory. Notoriously, also, it has been claimed that witnessing non-classicality in the gravitational field is practically impossible. Here, we propose a thought experiment that witnesses the non-classicality of a physical system by probing it with a qubit, without having to assume any specific dynamics on the physical system. Remarkably, this experiment does not require any quantum control of the system, involving only measuring a single classical observable on it. That non-classicality of a system can be established indirectly, by coupling it to a qubit, opens up exciting possibilities, for example, that quantum gravitational effects might be witnessed in the lab.

npj Quantum Information (2017)3:41 ; doi:10.1038/s41534-017-0040-4

\section{INTRODUCTION}

Whether all physical systems have quantum-like features is still a controversial issue. From time to time it has even been claimed that quantum theory's principles are not universally applicable: quantum features must disappear at a certain scale. ${ }^{1}$ The main problem with settling this issue is that it is hard to gather evidence for the quantisation of a given system. With the advent of better technologies, it has been possible to test the validity of quantum theory in an ever wider domain, towards more and more macroscopic quantum systems, but the problem is still open. In addition, whether the gravitational field is quantum is highly controversial. This is largely because direct evidence in favour of the quantisation of gravity is, to this day, hard to obtain. Despite the recent success in detecting gravitational waves, the detection of gravitons-quantum particles mediating the gravitational field - has been argued to be practically impossible. ${ }^{2,3}$

In this context, it is of essence being able to witness nonclassical features via methods that are as general as possible; in particular, they should assume as little as possible about the dynamics and features of the system whose non-classicality is to be tested.

Some indirect evidence of quantum-like features can be gathered by probing the system with a quantum system. To illustrate what "indirect" means, we can start with a thought experiment proposed by Feynman. ${ }^{4}$ In that experiment a test mass in a superposition of two different locations interacts with the gravitational field (see Fig. 1), but the same logic would be applicable to any other field.

Feynman's point was that the physical state of the composite system would have different properties according to whether the mass is entangled with gravity, or just somehow classically correlated with it. These two situations could in principle be distinguished, but this requires witnessing non-classicality in the gravitational field. However, the thought experiment seems to conceal a circularity-which appears to affect any such indirect witness. Witnessing the non-classicality of some system, such as the gravitational field, would seem to require either measuring two complementary observables on the system itself, or that it undergoes some interference process (which itself frequently constitutes a measurement of two complementary observables). However, the possibility of performing either of those operations is precisely what the thought experiment is designed to assessi.e., that the system in question (the field for Feynman's experiment) is quantum.

This is an instance of a more general problem, affecting the predictions of theoretical arguments in favour of quantisation of generic systems. Such arguments ${ }^{4-8}$ claim that a subsystem of the universe (e.g. a field) interacting with a quantum system (which here means any physical system that can implement a qubit) must itself be quantised. The problem is, again, that testing these predictions might seem to require full quantum control of the system that is argued to be quantised, but the existence of such a control is what the test is supposed to probe.

In this paper, we offer a solution to this problem. We propose a new thought experiment to witness the non-classicality of a system, by probing it via a qubit, without requiring any quantum control on the system. Specifically, the experiment is performed on the composite system of a qubit $S_{Q}$ and of a classical system $S_{C_{1}}$ which is assumed to have only a single observable $T$. By "classical" we mean precisely a system that has only one single observable. Our proposed test for non-classicality only requires to measure correlations involving just the observable $T$ on $S_{C}$ and the system $S_{C}$ need not undergo any interference. Appropriate values of these correlations, as we shall show, imply that the classical system $S_{C}$ must have at least another observable that cannot be simultaneously sharp when the observable $T$ is. This is our indirect witness of non-classicality. We then conjecture the possibility of an indirect measurement of the complementary observable $S$, by coupling the classical system with a qubit, via a teleportation-type scheme.

\footnotetext{
${ }^{1}$ Physics Department, University of Oxford, Oxford, UK and ${ }^{2}$ Centre for Quantum Technologies, National University of Singapore, Singapore, Singapore Correspondence: C. Marletto (chiara.marletto@gmail.com)
}

Received: 11 May 2017 Revised: 11 August 2017 Accepted: 14 August 2017

Published online: 05 October 2017 


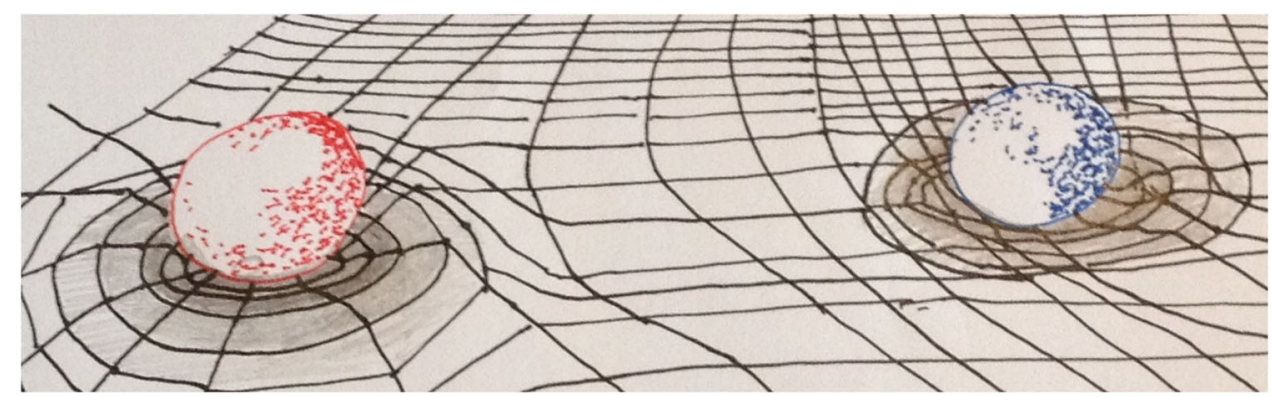

Fig. 1 An artist's impression of a mass in a superposition of two locations. In each of the two locations it interacts with the gravitational field, thus creating correlations. Feynman's thought experiment explores the issue of whether these correlations are classical or quantum-and it is generally applicable to any field

Remarkably, our thought experiment is formulated in a general, information-theoretic framework-which is independent of the details of the dynamics of the system $S_{c}$. Thus, our result is relevant within a wide range of different contexts: for example, it can be applicable to quantum gravity tests, and to testing the non-classicality of macroscopic systems, be they biological systems ${ }^{9,10}$ or computational devices. ${ }^{11}$ After all, in any experiment quantum control can only be assumed to exist on a limited number of degrees of freedom, while the rest could for all practical purposes be classical.

\section{RESULTS}

Let $\hat{q}^{(1)} \doteq\left(\sigma_{x} \otimes I, \sigma_{y} \otimes I, \sigma_{z} \otimes I\right)$ denote the vector of generators $q_{a}^{(1)}$ of the algebra of observables of the qubit $S_{Q}$, where $\sigma_{a}, a=x$, $y, z$, are the Pauli operators and $l$ is the single-qubit unit. Let $T$ be a binary observable on the classical system $S_{C}$ in other words, the classical system is supposed to be a single bit. Without loss of generality, we can represent it as an operator $q_{z}^{(2)} \doteq I \otimes \sigma_{z}$. For example, in the case of gravity, $T$ could be a discretised version of the position observable, representing two different locations of a mass which interacts with the quantum system through gravity. (If $S_{C}$ has a higher dimensionality, our result applies as long as one considers a quantum system $S_{Q}$ with the same dimensionality as $\left.S_{C}\right)$.

Consider now an operation defined so that it performs a classical copy of the values held by $S_{Q}$ with $S_{C}$ as the target, in the basis defined by the observable $q_{z}^{(1)} q_{z}^{(2)}$. In other words, in that basis, it is required to perform the computation $\{00 \rightarrow 00$, $10 \rightarrow 11\}$, where the first slot represents the value held by $S_{Q}$ and the second slot the value held by $S_{C}$. However, it is unknown what effect it has on other input states. This is because our scheme is independent of the details of the dynamics. The thought experiment precisely investigates what happens when the input states are $| \pm\rangle|0\rangle$, where $q_{1}^{(z)}$ is not sharp. Those states therefore act as a probe.

Note that the copy-operation is not assumed to be coherent, unitary or reversible in any sense. For example, it could be thought of as a classical controlled-NOT gate, where the NOT gate is realised by some classical evolution, such as $|0\rangle\langle 0| \rightarrow$ $\cos ^{2}(t)|0\rangle\left\langle 0\left|+\sin ^{2}(t)\right| 1\right\rangle\langle 1|$ for appropriate arguments $t$ (though the evolution need not be continuous).

The thought experiment goes as follows. First, prepare the qubit $S_{Q}$ in the eigenstates $| \pm\rangle$; of $| \pm\rangle$; and the classical system $S_{C}$ in some fixed state, which we denote as $|0\rangle$, representing a state where the observable $T$ is sharp with value 1 . Then, apply the copy-operation. Let us denote by $\rho_{ \pm}$the states of $S_{Q} \oplus S_{C}$ thus generated. At this point, measure the averages $\left\langle q_{a}^{(1)} q_{z}^{(2)}\right\rangle_{ \pm}, a=1$, 2,3 , and $\left\langle q_{z}^{(1)}\right\rangle_{ \pm}$on the states $\rho_{ \pm}$. Note that the global states $\rho_{ \pm}$of the composite system cannot be argued to be entangled by construction. This is because $S_{C}$ need not obey quantum theory.

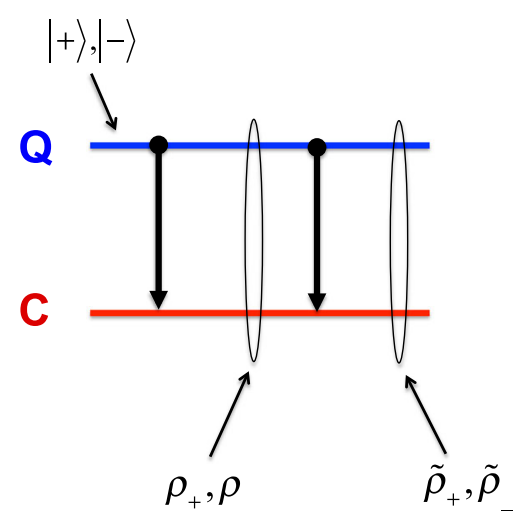

Fig. 2 Quantum network to prepare the states $\rho_{ \pm}$and $\tilde{\rho}_{ \pm} . Q$ denotes the quantum system, $C$ the classical system. The black arrow represents a copier in the basis defined by $q_{z}^{(1)} q_{z}^{(2)}$. No quantum control is required on $C$, as explained in the text

Now consider a different procedure, to prepare the states $\tilde{\rho}_{ \pm}$by applying the same copy-operation, as above, on each of the states $\rho_{ \pm}$. Then, measure $\left\langle A_{i}^{(1)} q_{z}^{(2)}\right\rangle_{\tilde{I}}$ for appropriate observables $A_{i}^{(1)} q_{z}^{(2)}$
(see Fig. 2).

We shall now argue that certain values of the correlation functions $\left\langle q_{a}^{(1)} q_{z}^{(2)}\right\rangle_{ \pm}$and $\left\langle A_{i}^{(1)} q_{z}^{(2)}\right\rangle_{\tilde{I}}$ imply that the classical system must have at least another observable that is complementary to $T$. Crucially, both correlation functions only require the "classical" observable $T$ to be measured on $S_{C}$.

Note first that, in our representation, the most general form of a state of $S_{Q} \oplus S_{C}$ is

$\rho=\frac{1}{4}\left(I+\underline{r} \cdot \hat{q}^{(1)}+s_{z} q_{z}^{(2)}+\underline{t} \cdot \hat{q}^{(1)} q_{z}^{(2)}\right)$

for some real-valued vectors $\underline{r}, \underline{t}$ and for some real coefficient $s_{z}$. This state, when interpreted as a two-qubit state, is separable and has no discord. ${ }^{12}$

Now, suppose that $\left\langle q_{z}^{(1)} q_{z}^{(2)}\right\rangle_{ \pm}=1$ and $\left\langle q_{a}^{(1)} q_{z}^{(2)}\right\rangle_{ \pm}=0, \forall a \neq z$, are observed, for both $\rho_{ \pm}$. This confirms that the quantum and the classical systems have undergone some interaction, because that value differs from the same correlation function evaluated for the initial states $| \pm\rangle|0\rangle$. Suppose also that $\rho_{ \pm}$are ensemble distinguishable from eigenstates of $q_{z}^{(1)} q_{z}^{(2)}$, by measuring $\left\langle q_{a}^{(1)}\right\rangle_{ \pm}$. This rules out the possibility that $\rho_{ \pm}$are themselves eigenstates in that basis. To satisfy these conditions, one must require $\quad \underline{r}=\underline{0}, \quad s_{Z}=0 \quad$ and $\underline{t}=(0,0,1)$. Thus, $\rho_{+}=\rho_{-}=\frac{1}{4}\left(I+q_{z}^{(1)} q_{z}^{(2)}\right)$. 
Suppose further that it is possible to find observables $A_{i}$ of the qubit with the property that measuring $\left\langle A_{i}^{(1)} q_{z}^{(2)}\right\rangle_{\tilde{ \pm}}$ can distinguish $\rho_{-}$. This implies that $\rho_{+} \neq \rho_{-}$, which is a contradiction.

Hence, we conclude that in order to reproduce the above correlation functions, the classical system must have an additional observable $T^{\prime}$ that cannot be simultaneously sharp when $T$ is. In our representation, that observable can be represented as an operator $q_{x}^{(2)}$, which does not commute with $q_{z}^{(2)}$. Our thought experiment therefore constitutes a witness of non-classicality on the system $S_{C}$ as promised.

\section{DISCUSSION}

This non-classicality could be more general than the strictly quantum one. For example, $T$ and $T^{\prime}$ might be two overlapping distributions in phase space, corresponding to uncertainty in preparation, as in Spekkens' toy model. ${ }^{13}$ This would effectively correspond to $S_{C}$ consisting of two classical bits whose values cannot be perfectly resolved. However, this model does not have a natural dynamics, so it is unclear what it would imply as to the physics of this thought experiment, or about the physical constitution of $S_{C}$.

Our proposed witness is also different from schemes based on non-contextuality and Kochen-Specher (KS) theorems. ${ }^{14}$ Testing the KS condition for non-contextuality relies on being able to perform projective measurements in a number of complementary observables on the system of interest, while our argument only assumes measurements of a single classical observable on the system $S_{C}$.

Violating Leggett-Garg ${ }^{15}$ inequalities is another scheme that might be useful contrasting with our scheme. That scheme, too, relies on repeatedly measuring a single observable on the system $S_{C}$, at different times. The violation of the Leggett-Garg inequalities implies that the system has been evolving under quantumlike evolutions in between the measurements. It might be interesting to see if a Leggett-Garg-type test on $S_{C}$ as prepared in our thought experiment would lead to violation of the Leggett-Garg inequalities. However, it could be that since the evolution of $S_{C}$ via the coupling with the quantum system induces decoherence, no violation occurs. Our scheme instead relies on measuring correlations between the system $S_{C}$ and the quantum system used to as a probe. In which case, our scheme is a more suitable option for detecting non-classicality.

Crucially, our test only requires applying the copy-operation in the basis defined by $T$, in order to prepare the relevant states, and that those states can be discriminated by ensemble measurements, realised by measuring the local observables $A_{i}$ on the qubit and just $T$ on the $S_{C}$. These states could, in particular, be not perfectly distinguishable via single-shot measurements (thus the overall evolution might be non-unitary). Therefore, our thought experiment does not presuppose the possibility of performing any interference on the classical system, nor the possibility of measuring other observables than $T$.

Whilst this test allows one to conclude that there must be an additional observable $T^{\prime}$, which is necessary to describe the accessible states of $S_{C}$, that observable need not be measurable directly. It might be, for example, that there is some fundamental limitation to how well its eigenstates can be resolved from one another: this highlights an interesting distinction between an observable being directly measurable and its being necessary to describe the accessible states of a system.

We now discuss how assuming the possibility of a coherent interaction between the classical system and some other qubit allows one to measure indirectly that observable. The scheme goes as follows.

As before, prepare the states $\rho_{ \pm}$on $S_{Q} \oplus S_{C}$. At this point, apply the operation, which in the basis defined by $q_{z}^{(1)} q_{z}^{(2)}$ realises the computation $\{00 \rightarrow 00,01 \rightarrow 11\}$. This is a copy of the $q_{z}$ values

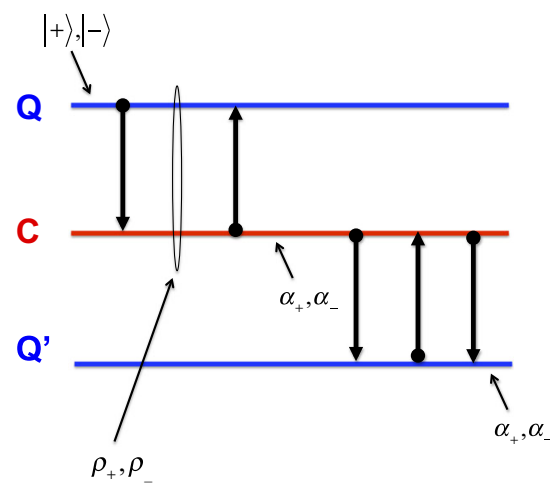

Fig. 3 Quantum network to prepare the states $a_{ \pm}$on the classical system $C . Q^{\prime}$ represents the second qubit where the states $a_{ \pm}$are accessible. Again, no quantum control is required on the system $C$ (see explanation in the text)

held by $S_{C}$, this time with $S_{Q}$ as the target. As explained in ref. 16, this operation must generate, acting on each of the states $\rho_{ \pm}$, two new (possibly mixed) states $a_{+}$and $a_{-}$on $S_{C}$. As we said, these states need not be distinguishable from one another; moreover, since it is not possible to measure any other observable than $T$ on $S_{C}$, one cannot reconstruct them by applying a procedure such as state tomography directly to $S_{C}$. However, by bringing in another qubit $S_{Q^{\prime}}^{\prime}$ it is possible to apply on $S_{C} \oplus S_{Q}^{\prime}$ a sequence of three CNOT gates in the $q_{z}$ basis to perform a logical swap. ${ }^{17}$ This allows one to prepare the qubit $S_{Q}^{\prime}$ in each of those two states. At this point, state tomography on the qubit allows one to distinguish the states asymptotically, thus showing indirectly that those states existed on $S_{C}$ (see Fig. 3).

Incidentally, if the states $a_{+}$and $a_{-}$are not orthogonal to one another, an orthogonalisation procedure would allow one to argue for the existence of two other observables of $S_{C_{1}}$ in addition to $T$. Each one observable would be represented by an operator having, respectively, $a_{+}$and $a_{-}$as eigenstates. In the case of this test too, the only observable measured on the classical system is $T$; however, overall coherence is required to realise the swap.

We emphasise that the central feature of our analysis is that, it does not assume any particular dynamical model for the system, whose non-classicality is to be witnessed, nor for the coupling between it and the quantum system. This is in contrast to the recent argument in ref. 18, where quantumness of a given system is confirmed indirectly by its ability to entangle two other bona fide quantum systems. In that sense the argument in ref. 18 is an extension and elaboration of Feynman's argument. ${ }^{4}$ In the latter the witness of non-classicality would be the existence of bipartite entangled states generated by coherent evolution, while in ref. 18 the witness is the existence of states with discord between the classical and the quantum systems, which forces the classical system to have a complementary observable. In both these arguments, however, the interactions are all assumed to be unitary and, furthermore, the system to be tested is described within the same formalism; whereas in our scheme, crucially, there are no such assumptions.

Our thought experiment is also of practical importance as it illustrates how to manipulate quantumly a system on which there is no direct quantum control. Thus, our thought experiment is related to the experiments that prepare and subsequently witness "Schrödinger cat" states of light. ${ }^{19}$ There, the system $S_{C}$ corresponds to the EM field, initially prepared in a coherent state; $S_{Q}$ is an atom, initially prepared in a superposition of its two energy levels. The two systems interact in such a way that they evolve into an entangled state, which corresponds to one of our $\rho_{ \pm}$. By measuring the atom in a complementary basis, the field is left in one of two orthogonal Schrödinger cat states. The atom (or an ensemble of 
them) is then also used as a probe, to witness the cat states. In this way the complementary observables of the EM field are never measured directly, just like in our thought experiment.

Witnessing non-classicality of a physical system that need not obey quantum theory, is a key task in contemporary physics. It is crucial for testing predictions that gravity is quantised, but also to explore the quantum-to-classical boundary. For example, it is necessary to test predictions that macroscopic systems (e.g. a bacterium), coupled to a quantum system are, themselves quantum. In all such cases, one cannot assume full quantum control on the physical system $S_{C}$ whose non-classicality is to be witnessed. For instance, tests of non-classicality designed for quantum systems, e.g. violation of Bell inequalities, are inadequate. Our thought experiment is a proposal for a new approach to performing that task. Its strength is that, by using a quantum probe, it provides an indirect witness of non-classicality, which requires only measuring a single observable on the system $S_{C}$. In addition, it is remarkably general: it only relies on informationtheoretic witnesses, without assuming any particular dynamics for the system $S_{C}$. Thus, our experiment is applicable to all the above open problems-a task that we leave for future work.

Data availability

This work has no data.

\section{ACKNOWLEDGEMENTS}

The authors thank Tomek Paterek and Tanjung Krisnanda for helpful comments, and two anonymous referees for suggesting useful improvements. C.M.'s research was supported by the Templeton World Charity Foundation. V.V. thanks the Oxford Martin School, the John Templeton Foundation, the EPSRC (UK) and the Ministry of Manpower (Singapore). This research is also supported by the National Research Foundation, Prime Ministers Office, Singapore, under its Competitive Research Programme (CRP Award No. NRF- CRP14-2014-02) and administered by Centre for Quantum Technologies, National University of Singapore.

\section{AUTHOR CONTRIBUTIONS}

Both authors researched, collated, and wrote this paper.

\section{ADDITIONAL INFORMATION}

Competing interests: The authors declare no competing financial interests.

Publisher's note: Springer Nature remains neutral with regard to jurisdictional claims in published maps and institutional affiliations.

\section{REFERENCES}

1. Penrose, R. On Gravity's role in quantum state reduction. General Relativ. Gravit. 28, 581-600 (1996).
2. Dyson, F. Is a graviton detectable? Int. J. Mod. Phys. A 28 (2012).

3. Rothman, T. \& Boughn, S. Can gravitons be detected? Found. Phys. 36, 1801 (2006).

4. Feynman, R. Chapel Hill Conference Proceedings. http://www.edition-opensources. org/sources/5/1/index.html (1957).

5. De Witt, B. A Global Approach to Quantum Field Theory. International Series of Monographs on Physics (Clarendon Press, 2014).

6. Peres, A. \& Terno, D. Hybrid classical-quantum dynamics. Phys. Rev. A 63 (2001).

7. Heinsenberg, W. Physical Principles of the Quantum Theory. Dover Books on Physics (Dover Publications, Inc., 1998).

8. Bohr, N. \& Rosenfeld, L. Kgl. Danske Vidensk. Selsk. Mat. Fys. Med. 12, 8 (1933).

9. Coles, D. M. et al. Living polaritons: modifying energy landscapes in photosynthetic organisms using a photonic structure. Preprint at arXiv:1702.01705 (2017).

10. Marletto, C., Coles, D. M., Farrow, T. \& Vedral, V. Entanglement between living bacteria and quantized light witnessed by Rabi splitting. Preprint at arXiv:1702.08075 (2017).

11. Boixo, S. et al. Quantum annealing with more than one hundred qubits. Nat. Phys. 10, 218224 (2013).

12. Modi, K., Brodutch, A., Cable, H., Paterek, T. \& Vedral, V. The classical-quantum boundary for correlations: discord and related measures. Rev. Mod. Phys. 84, 1655 (2012).

13. Spekkens, R. Evidence for the epistemic view of quantum states: a toy theory. Phys. Rev. A 75, 032110. https://link.aps.org/doi/10.1103/PhysRevA.75.032110 (2007).

14. Kochen, S. \& Specker, E. P. The problem of hidden variables in quantum mechanics. J. Math. Mech. 17, 59-87 (1967).

15. Leggett, A. J. \& Garg, A. The Problem of Hidden Variables in Quantum Mechanics. Phys. Rev. Lett. 54, 857 (1985).

16. Marletto, C. \& Vedral, V. Why we need to quantise everything, including gravity. Preprint at https://arXiv.org/pdf/ 1703.04325 (2017).

17. Nielsen, M., Chuang, R. Quantum Information and Quantum Computation (CUP, 2000).

18. Krisnanda, T., Zuppardo, M., Paternostro, M. \& Paterek, T. Revealing non-classicality of unmeasured objects. Preprint at http://arxiv.org/abs/ 1607.01140.

19. Haroche, S., Brune, M. \& Raymond, R. Manipulation of photons in a cavity by dispersive atom-field coupling: quantum-nondemolition measurements and generation of "Schrodinger cat" states. Phys. Rev. A 45, 7 (1992).

(c) Open Access This article is licensed under a Creative Commons Attribution 4.0 International License, which permits use, sharing, adaptation, distribution and reproduction in any medium or format, as long as you give appropriate credit to the original author(s) and the source, provide a link to the Creative Commons license, and indicate if changes were made. The images or other third party material in this article are included in the article's Creative Commons license, unless indicated otherwise in a credit line to the material. If material is not included in the article's Creative Commons license and your intended use is not permitted by statutory regulation or exceeds the permitted use, you will need to obtain permission directly from the copyright holder. To view a copy of this license, visit http://creativecommons. org/licenses/by/4.0/.

(c) The Author(s) 2017 